\title{
Re-assessment of chronic radio-induced tissue damage in a rat hindlimb model
}

\author{
BÉRENGÈRE PHULPIN ${ }^{1,2}$, GILLES DOLIVET ${ }^{1}$, PIERRE-YVES MARIE ${ }^{5}$, SYLVAIN POUSSIER $^{5}$, \\ PATRICE GALLET ${ }^{1}$, AGNÈS LEROUX ${ }^{3}$, PIERRE GRAFF ${ }^{4}$, FREDERIQUE GROUBACH ${ }^{6}$, \\ PIERRE BRAVETTI ${ }^{7}$, JEAN-LOUIS MERLIN $^{2}$ and NGUYEN TRAN ${ }^{5,6}$ \\ ${ }^{1}$ Head and Neck and Dental Surgery Units, Oncologic Surgery Department, Centre Alexis Vautrin; \\ ${ }^{2}$ Tumor Biology Unit, EA4421 SIGReTO UHP-Nancy University, Centre Alexis Vautrin; \\ Departments of ${ }^{3}$ Pathology, and ${ }^{4}$ Radiotherapy, Centre Alexis Vautrin, 54511 Vandoeuvre-lès-Nancy; \\ ${ }^{5}$ INSERM-U961, and ${ }^{6}$ School of Surgery, Faculty of Medicine, UHP-Nancy University, 54500 Vandoeuvre-lès-Nancy; \\ ${ }^{7}$ Department of Oral Surgery, Faculty of Dentistry, University of Nancy, 54004 Nancy, France
}

Received March 16, 2010; Accepted May 17, 2010

DOI: $10.3892 /$ etm_00000087

\begin{abstract}
Radiotherapy is successfully used to treat neoplastic lesions, but may adversely affect normal tissues within the irradiated volume. However, additional clinical and para-clinical data are required for a comprehensive understanding of the pathogenesis of this damage. We assessed a rat model using clinical records and medical imaging to gain a better understanding of irradiation-induced tissue damage. The hindlimbs of the rats in this model were irradiated with a single dose of 30 or $50 \mathrm{~Gy}$. Sequential analysis was based on observation records of stage and planar scintigraphy. Additional radiography, radiohistology and histology studies were performed to detect histological alterations. All animals developed acute and late effects, with an increased severity after a dose of $50 \mathrm{~Gy}$. The bone uptake of ${ }^{99} \mathrm{~m}$ Tc-HDP was significantly decreased in a dose- and time-dependent manner. Histologically, significant tissue damage was observed. After the 50 Gy irradiation, the animals developed lesions characteristic of osteoradionecrosis (ORN). Radiographic and histological studies provided evidence of lytic bone lesions. Our rat model developed tissue damage characteristic of radiation injury after a single $30 \mathrm{~Gy}$ irradiation and tissue degeneration similar to that which occurs during human ORN after a 50 Gy irradiation. The development of this animal model is an essential step in exploring the pathogenesis of irradiation-induced tissue damage, and may be used to test the efficacy of new treatments.
\end{abstract}

Correspondence to: Dr Bérengère Phulpin, Head and Neck and Dental Surgery Units, Oncologic Surgery Department, Centre Alexis Vautrin, Avenue de Bourgogne, Brabois, 54511 Vandoeuvrelès-Nancy, France

E-mail: b.phulpin@nancy.fnclcc.fr

Key words: radiotherapy, osteoradionecrosis, rat model, scintigraphy, radiohistology

\section{Introduction}

Radiotherapy is successfully used to treat regional neoplastic lesions, but may have an adverse effect on normal tissues. Irradiated tissue abnormalities include the impairment of vascularization (1-3) due to the high vulnerability of small vascular endothelial cells, impairment of cell homeostasis with cellular apoptosis (3-6), and the accumulation of fibrosis $(5,7)$. Bone tissue is very vulnerable to irradiation $(8,9)$ and undergoes impaired healing, infection, atrophy, pathological fractures and bone tissue necrosis, termed osteoradionecrosis (ORN), within the irradiated region. These iatrogenic delayed complications occur in various anatomic sites, including the pelvis, sternum, vertebrae, clavicle, femoral head and, in particular, the mandible. The most devastating radiotherapyinduced complications of the head and the neck occur in the mandible, and in some cases require surgical resection (10). The reported incidence of ORN following conventional radiotherapy (RT) to the mandible ranges between 0.9 and $35 \%$ (11), with an increased risk at doses exceeding 60 Gy (12).

Due to the increased use of radiation therapy alone or in combination with chemotherapy in the treatment of head and neck malignancies $(13,14)$, it is likely that the number of radio-induced complications will rise (14-16).

Despite increased interest in the clinical development of ORN along with a new proposed definition and classification (17), most animal model studies on ORN date from the 1960-70s (18-20). Since then, imaging techniques have evolved, and the renewed investigation of treatments for ORN in animal models is warranted.

The aim of the present study was to develop an experimental animal model of hindlimb radiation-induced tissue damage in order to gain a better understanding of irradiation-induced defects and to determine potential targets for therapeutic strategies. We conducted a 10-month study in which rats were irradiated bilaterally on the hindlimbs with a single dose of 30 or $50 \mathrm{~Gy}$. Sequential analysis was based on observation records of stage and non-invasive imaging using planar scin- 

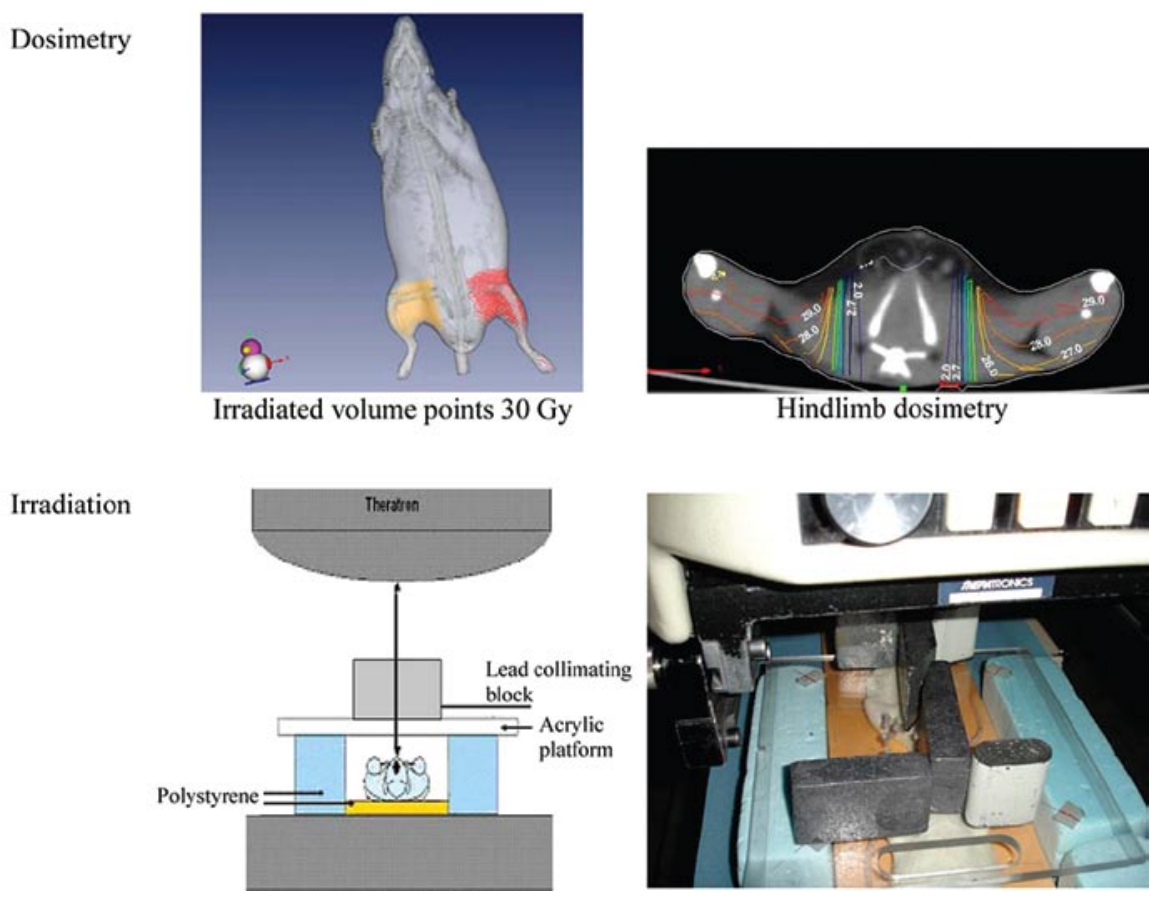

Figure 1. The 30-Gy irradiation procedure.

tigraphy techniques, focusing on changes in bone perfusion and bone lysis. Additional radiography, radiohistology and histology studies were performed to detect tissue alterations.

\section{Materials and methods}

Animals. This 10-month study was conducted using 24 adult male Wistar rats (Janvier CERJ, Le Genest Saint Isle, France) with an initial body weight of 420-460 g. The rats were maintained in a specific environment with controlled temperature and humidity and an automatically regulated 12-h light/dark cycle, and were fed a standard commercial diet and given water ad libitum. The experimental protocol was conducted in accordance with the regulations of our local ethics committee and with the Animal Welfare Act of the National Institutes of Health Guide for the Care and Use of Laboratory Animals (NIH Publication no. 85-23, Revised 1996). Subcutaneous injections of bupremorphine (Vetarsic ${ }^{\circledR} 0.5 \mathrm{mg} / \mathrm{kg}$ ) in association with intravenous injections of paracetamol (Perfalgan ${ }^{\circledR}$ $0.3 \mathrm{ml}$ ) were administered to the rats twice a week during the ORN phase.

General experimental design. Under general anesthesia, animals were administered a single dose of 30 Gy (Group 2, n=8) or 50 Gy (Group 3, n=8) bilaterally on the hindlimb. Results were compared to healthy control animals (Group 1).

Irradiation procedures. Cobalt 60 was chosen to minimize the difference between the absorbed dose in soft and hard tissues. Prior to irradiation, a scanner acquisition (Philips Brillance 40) enabled the calculation of dosimetry (Isogray 3.0 software Dosisoft). Irradiation of the hindlimb was performed under general anesthesia as described previously (21). Briefly, the animals were placed in a prone position upon a thick polystyrene phantom. The hindlimbs were additionally immobilized by adhesive tape. The skin distance focus was $70 \mathrm{~cm}$, and the field size was $20 \times 30 \mathrm{~cm}$. The lead collimating block was positioned on a $0.5-\mathrm{cm}$ thick acrylic platform to shield the body, allowing exposure of only the hindlimb without the pelvis. Radiation was delivered in a vertical beam from a Theratron ${ }^{\circledR}$ 780C X-ray machine delivering $\gamma$-rays of $1.25 \mathrm{MeV}$ energy. The irradiated volume was $40 \mathrm{~cm}^{3}$ at a dose rate of $1.4 \mathrm{~Gy} / \mathrm{min}$ (Fig. 1). The room temperature during irradiation was $22^{\circ} \mathrm{C}$.

Determination of stage. Since standardized staging of ORN is not available for animal models, our macroscopic determinations for the rat model were partly based on the development of human symptoms as described elsewhere (15). Rats were followed up weekly. Acute parameters of irradiation were the appearance of alopecia and irradiation-induced dermatitis. Chronic effects included foot edema and necrosis noted on the sole of the foot, later extending to the entire foot.

Planar scintigraphy. Bone uptake was assessed using ${ }^{99 \mathrm{~m} T c-H D P}$. Briefly, following the intravenous injection of $9 \mathrm{mCi}$ 99mTC-HDP, uptake was recorded under general anesthesia during the first $5 \mathrm{~min}$ for the measurement of blood flow time and $3 \mathrm{~h}$ later, representing the late static phase of bone uptake. A single-head gamma camera (Sopha DSX, SMV-GE) was used, equipped with a 1.5-mm pinhole collimator (165-mm focal length, 44-mm radius of rotation) with the following parameters: 256x256 matrix, 1.14 zoom and $140( \pm 20 \%) \mathrm{keV}$ energy window. The total acquisition time was $30 \mathrm{~min}$ : $15 \mathrm{~min}$ for ventral and $15 \mathrm{~min}$ for dorsal acquisition.

Changes in the accumulation of the tracer in the irradiated bone and surrounding tissues were evaluated by measuring uptake in regions of interest (ROIs) on Dysplay image processing computer software (Console Vision; General Electric $\left.^{\circledR}\right)$. 
Table I. Effect of irradiation doses on clinical changes.

\begin{tabular}{|c|c|c|c|c|c|c|}
\hline Rats & $\begin{array}{l}\text { Beginning } \\
\text { alopecia } \\
\text { (weeks) }\end{array}$ & $\begin{array}{c}\text { Radiation } \\
\text { dermatitis } \\
\text { (weeks) }\end{array}$ & $\begin{array}{c}\text { Total } \\
\text { alopecia } \\
\text { (weeks) }\end{array}$ & $\begin{array}{c}\text { Foot } \\
\text { edema } \\
\text { (weeks) }\end{array}$ & $\begin{array}{l}\text { Beginning necrosis } \\
\text { (sole of the foot) } \\
\text { (weeks) }\end{array}$ & $\begin{array}{l}\text { Beginning necrosis } \\
\text { (entire foot) } \\
\text { (weeks) }\end{array}$ \\
\hline \multicolumn{7}{|l|}{30 Gy } \\
\hline No. of cases & $8 / 8$ & $0 / 8$ & $0 / 8$ & $0 / 8$ & $0 / 8$ & $0 / 8$ \\
\hline Hindlimb & $16 / 16$ & $0 / 16$ & $0 / 16$ & $0 / 16$ & $0 / 16$ & $0 / 16$ \\
\hline Percentage & 100 & 0 & 0 & 0 & 0 & 0 \\
\hline Mean time of appearance & 6 & 0 & 0 & 0 & 0 & 0 \\
\hline Standard deviation & 2.1 & 0 & 0 & 0 & 0 & 0 \\
\hline \multicolumn{7}{|l|}{$50 \mathrm{~Gy}$} \\
\hline No. of cases & $7 / 7$ & $7 / 7$ & $7 / 7$ & $7 / 7$ & $6 / 6$ & $4 / 6$ \\
\hline Hindlimb & $14 / 14$ & $14 / 14$ & $14 / 14$ & $14 / 14$ & $11 / 12$ & $7 / 12$ \\
\hline Percentage & 100 & 100 & 100 & 100 & 91.67 & 58.33 \\
\hline Mean time of appearance & 2.86 & 6.36 & 7.57 & 16.17 & 25.82 & 27.71 \\
\hline Standard deviation & 0.24 & 0.74 & 2.06 & 8.08 & 13.41 & 11.59 \\
\hline
\end{tabular}

Radiographic studies. Radiographs of the feet and tibia were conducted on Roetgen film (Kodak InSight film, 31x41 mm) with a Kodak 2200 Intraoral X-ray System $(70$ kV, 7 mA and $0.138 \mathrm{sec}$ ) before the animals were sacrificed.

$99 \mathrm{~m}$ Tc bone activity was observed in a histological section and compared to previous results determined in vivo by scintigraphy.

Autoradiography and histology. In some animals, the tibia was excised and snap-frozen in liquid nitrogen-cooled isopentane. A $15-\mu \mathrm{m}$ cryosection, oriented along the vertical long axis of the bone, was performed. This section was analyzed on a beta micro-imaging system ( $\mu$ IMAGER; Biospace, Paris, France), which allows the recording of high resolution images in order to highlight the bone uptake of ${ }^{99 \mathrm{~m} T c}$. This imaging and counting system detects electrons emitted on the overall surface of histopathological slices with a high spatial resolution $(20 \mu \mathrm{m})(21)$.

Histology. Gastrocnemius muscle, tibias and feet were removed and fixed in AFA (acid acetic, formaldehyde and alcohol). Tibia and feet were decalcified in a solution of $70 \%$ ethanol and concentrated nitric acid for 2 days, then each section was embedded in paraffin, cut into $5-\mu \mathrm{m}$ sections and stained with H\&E prior to light microscopic observations.

Statistics. Quantitative data based on the ${ }^{99 \mathrm{~m} T c}$ activity of the ROIs, namely the hindlimb, knees and feet, relative to the chest and corrected to background, were expressed as the mean \pm SD. The Student's t-test was used for pairwise comparisons. A p-value of $<0.05$ was considered to be indicative of a significant difference.

\section{Results}

Effect of irradiation on macroscopic staging recordings. The weight of the animals steadily increased $(440 \pm 20 \mathrm{~g}$ before irradiation, $519 \pm 68 \mathrm{~g}$ at 6 months and $590 \pm 79 \mathrm{~g}$ at 10 months after irradiation), as compared to the reference weight curve provided by the animal provider $(463 \pm 80 \mathrm{~g}$ at 6 months and $550 \pm 92 \mathrm{~g}$ at 10 months).

The observed pathological characteristics of the animals are listed in Table I. All irradiated animals developed alopecia within the irradiated area. In the animals of Group 2 (30 Gy), alopecia became apparent in the irradiated hindlimb on average 4-5 weeks after irradiation, and was visible and irreversibly stabilized at 8-10 weeks (Fig. 2). In the group of rats receiving a single dose of 50 Gy (Group 3), 1 rat died 1 week after irradiation. Alopecia appeared early (2-3 weeks after irradiation) and hair loss was total at $5 \pm 2$ weeks. Thereafter, the irradiation-induced skin lesions gradually intensified, evolving from dry to moist desquamation. After an average of 13 weeks, significant edema of the toes was observed, which spread to the entire foot after 16 weeks. There was a gradual loss of elasticity of the irradiated hindlimb. After anesthesia, one rat was euthanized (with an intravenous injection of an overdose of sodium pentobarbital; Ceva Santé Animale ${ }^{\circledR}$ ) at 24 weeks post-irradiation due to a large area of skin necrosis on the right thigh. After $25 \pm 13$ weeks, small areas of necrosis began to be visible on the soles of the feet of the remaining 6 animals (11/12 feet). The necrosis was found to be very aggressive and expanded rapidly to the full-face plantar. After 26 weeks, atrophy of the toes was observed in 6 feet and was further characterized by the formation of scindactyly and by the appearance of a dry and black necrosis along with the disappearance of phalanges of the toe. After 29 weeks, necrosis encompassed the entire foot and progressed to the hindlimb (Fig. 2).

Development of bone perfusion by sequential planar scintigraphy. Quantitative analysis of planar SPECT imaging (Fig. 3A) revealed that the early uptake of ${ }^{99 m}$ Tc-HDP, i.e., blood flow activity, did not differ between animals which received 30 Gy of irradiation compared to the controls, irrespective of the SPECT exams recorded at 2 or 8 months after irradiation $(13 \pm 2.76$ and $12 \pm 1.38 \%$, respectively) (Fig. 4A). By contrast, 


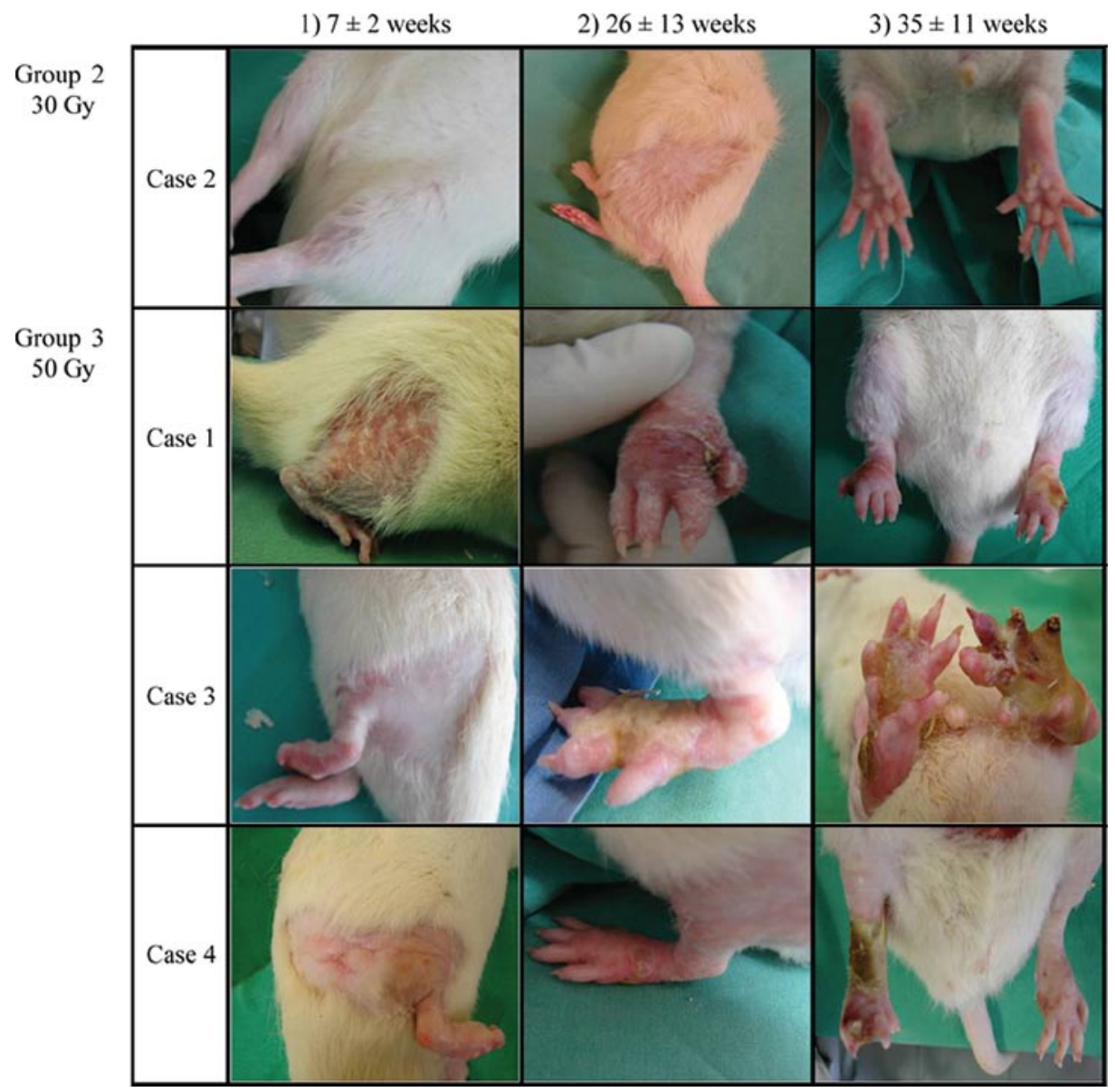

Figure 2. Images showing symptomatic changes. In Group 2, the animals presented a partial and persistent alopecia. In Group 3, alopecia was initially observed, and rapidly developed into a total irreversible alopecia. No further symptoms were observed in the 30-Gy irradiated animals. In Group 3, edema and necrosis appeared in the irradiated rat feet and progressed to the hindlimbs, affecting both bone and soft tissue.

A
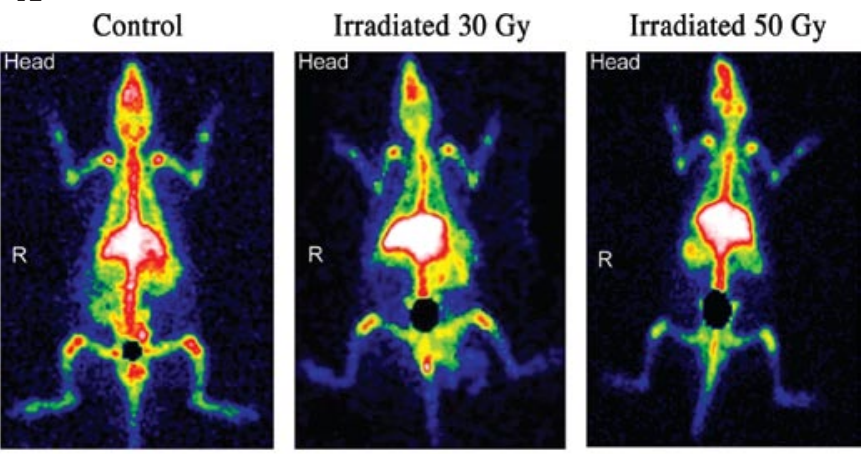

B

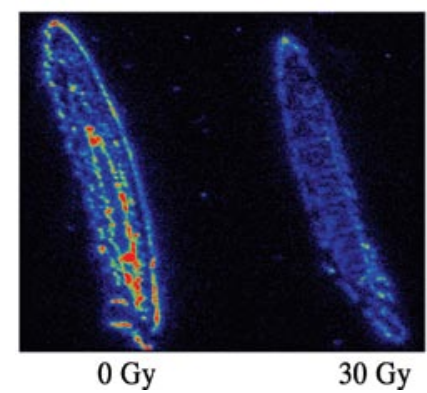

Figure 3. (A) Representative scintigraphic images highlighting changes in the uptake of ${ }^{99 \mathrm{~m} T C-H D P}$ in 30- and 50-Gy irradiated bone. (B) Representative $\mu$ IMAGER images confirming a decrease in the bone tracer after 2 months. a significant decrease in blood flow with ischemic hindlimb was observed at 2 months in animals that received $50 \mathrm{~Gy}$ in comparison to the controls $(-17.51 \%, \mathrm{p}<0.05)$. At 8 months post-irradiation, there was a $72 \%$ increase in early ${ }^{99 \mathrm{~m}} \mathrm{Tc}-\mathrm{HDP}$ uptake in this group compared to the controls, suggesting a possible contribution of inflammatory conditions.

The bone uptake of ${ }^{99 \mathrm{~m} T c-H D P}$ was found to be significantly decreased in the 30-Gy (Fig. 3B) and 50-Gy groups. In the 30-Gy group, a time-dependent decrease in bone uptake was observed with $-18 \%$ at 2 months and $-29 \%$ at 8 months compared to the normal hindlimb. In the 50-Gy group, the decrease in the mean bone uptake was already maximal at 2 months (-31\%), and stabilized at 8 months (-21\%) (Fig. 4B).

Radiographic and autoradiographic studies. In 5 rats prior to sacrifice, foot radiographs highlighted bone lysis characterized by a loss of one or two distal and middle toe phalanges (Fig. 5). Patchy bone foot mineralization was observed, with the presence of blurring trabecular bone. Moreover, there was a thinning of the distal extremity of the posterior tibia cortical and a slight thickening of the tibia cortical.

Histology. Irradiation of the rat hindlimb at a dose of $30 \mathrm{~Gy}$ produced a decrease in vascularization and a partial loss of osteocytes (Fig. 6). By contrast, irradiation at a single dose of 50 Gy induced dramatical changes in the overall architecture 
A

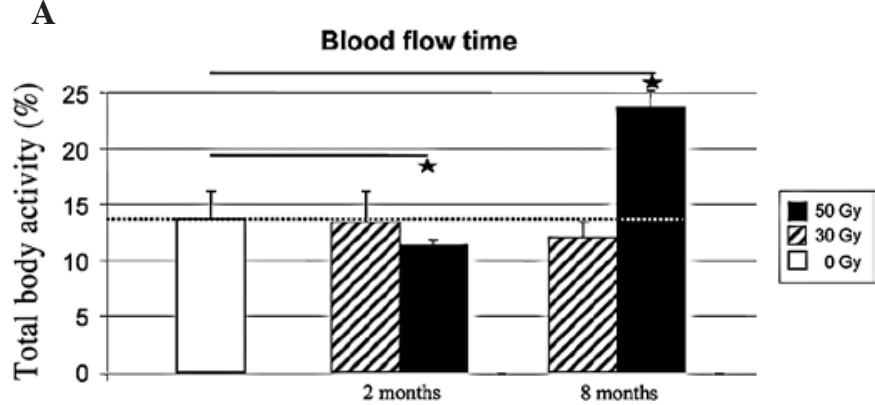

B

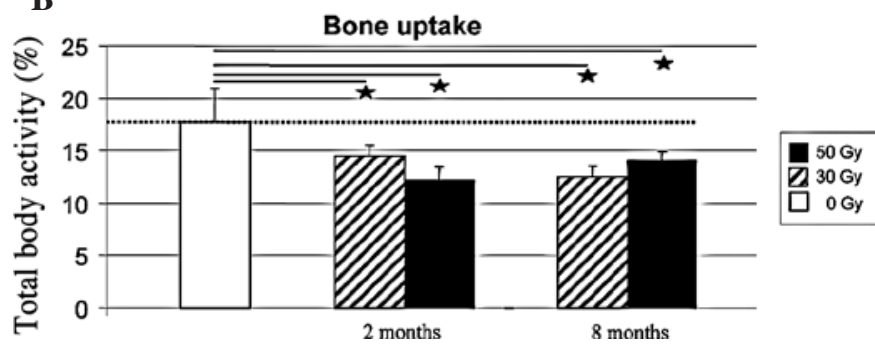

Figure 4. Progression of blood flow time (A) and bone uptake (B) in the rat hindlimb after 30-Gy (hatched columns) and 50-Gy (black columns) irradiation determined by ${ }^{99 \mathrm{~m}} \mathrm{Tc}$ activity.

of the bone and soft tissues of the hindlimb. Acellular bone was observed and characterized by a loss of osteocytes on the cortical bone and osteoblasts from bone margins resulting in empty Howship's lacunae. In addition, a detachment or separation of the periosteum was noted, and blood vessels of the periosteum were destroyed and replaced by fibrosis. In the bone marrow tissue, an almost total loss of hematopoietic cells was found. These were replaced by adipocytes along with an amorphous eosinophil substance (Fig. 6A). Blood vessels that supply the bone marrow were surrounded by fibrosis. Typically, these vessels were characterized by a disappearance of endothelial cells, a collagenous hyalinization of the blood vessel wall and a narrowing of the vascular lumen (Fig. 6B). Abnormal bone resorption along with an increase in the osteoclast population within the resorption cavities was also observed.

At the level of the foot skin, the epidermis was very thick with hypertrophic cells containing dystrophic nuclei. Necrosis occurred in the skin causing interruptions in the epithelium in the skin ulcers. The dermis was loose, and almost all of the cells were dead as no nucleus was visible in the histological section. There were areas of inflammatory infiltration (lymphocyte and mast cells), with the tissues being edematous and necrotic.

At necropsy, the gastrocnemius muscle was adherent to the surrounding tissues. Histologically, muscle destruction was also evidenced by an increase in the collagen component and in inflammatory infiltration (lymphocyte and mast cells) between the fibers. The presence of few fibro-necrotic areas was noted.

Two rats in Group 3 experienced 3 neoplasias. In the first case, one tumor developed 7 months after the onset of irradiation in an area of anterior radiodermatitis. There was ulceration on the right thigh with a keratinous crust, surrounded by elevated and indurated borders. The surrounding skin

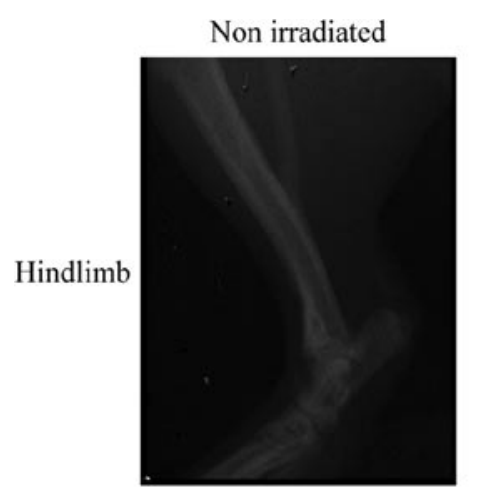

Osteoradionecrosis
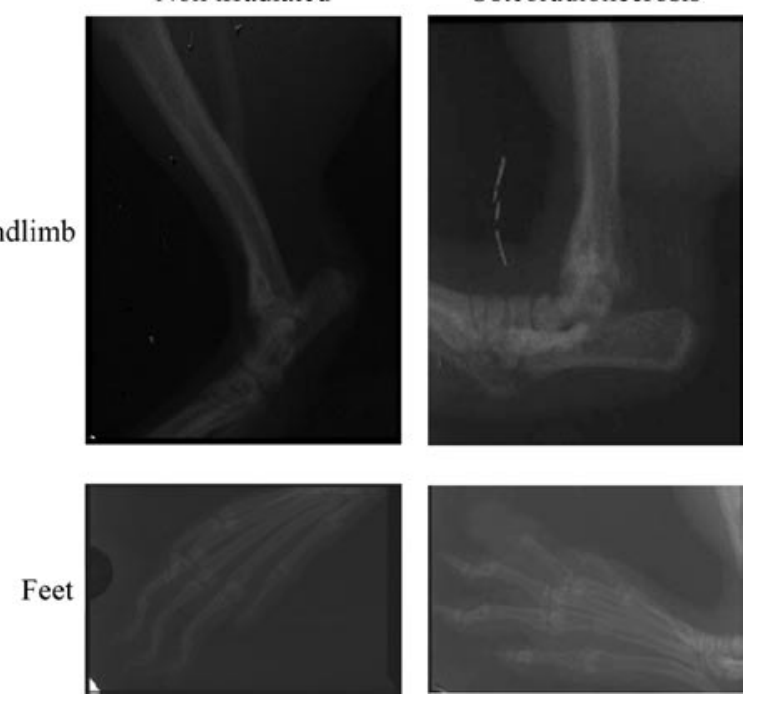

Figure 5. Examples of radiography showing cortical thickening of the tibia and foot bone lysis.

appeared affected, and the tumor showed rapid enlargement. Histological studies revealed that it was a squamous cell carcinoma. In the other case, 2 neoplasias became apparent 10 months after irradiation, one on each thigh. The tumors were well-circumscribed, located deep within the soft tissues and firm at palpation. They were associated with necrotic ulceration, located at the surface of the skin and surrounded by elevated and indurated borders. Both tumors were very expansive and histologically characterized as soft tissue sarcomas (Fig. 7).

\section{Discussion}

The long-term effects of irradiation such as osteoradionecrosis (ORN) persist for months or years after exposure. Therefore, an understanding of radiation-related damage and the development of novel therapies to treat acute side effects as well as late events are crucial. The current study conducted macroscopic observation of staging and analyzed scintigraphic and histological changes in a rat hindlimb model exposed to several single doses of irradiation. Overall, the data suggest that the long-term effects of radiation, particularly at high doses, are associated with the severe development of ORN, including i) a dramatic alteration in the structural integrity of both soft and hard tissue, accompanied by ii) abnormal bone perfusion and, in some instances, iii) the development of irradiation-induced tumors.

Several studies have addressed the question of whether there are dose- and time-dependent relationships with irradiation in various soft and hard tissues in human or animal models $(1,8,15,17,22)$. Most studies have demonstrated complex alterations following iatrogenic irradiation in experimental models (7,23), and it is generally considered that dose and irradiated volume are adequate predictors of the probability of complications (7). However, the development of ORN and its qualitative or quantitative aspects, particularly on a longitudinal basis, remain scarcely understood. 
A

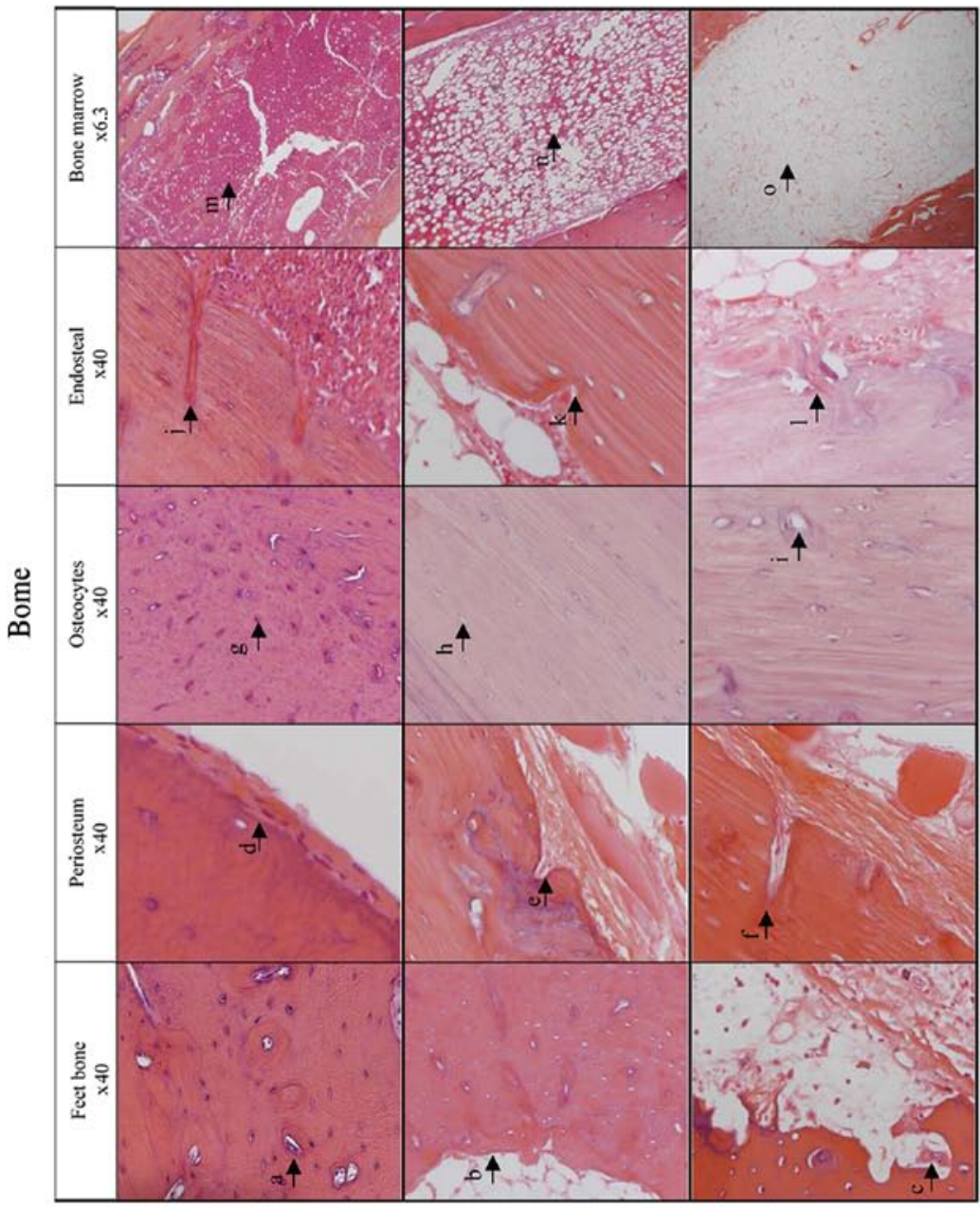

B
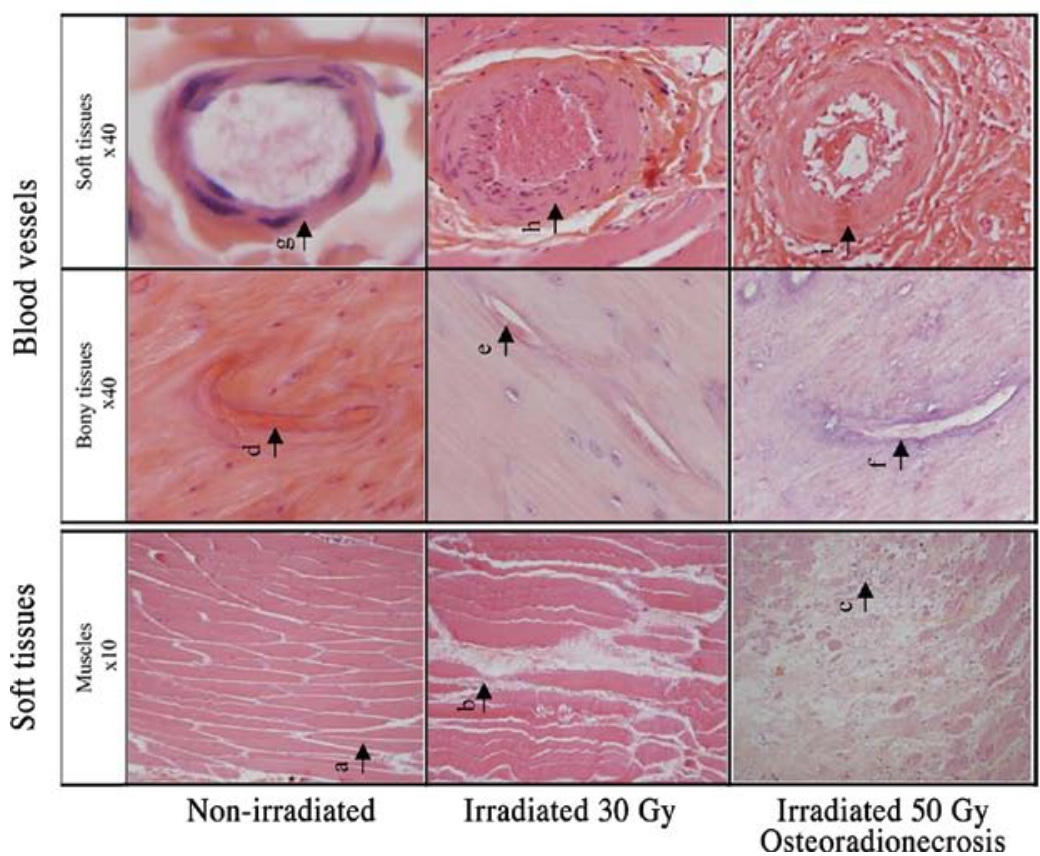

Figure 6. Micrographs of normal, 30- and 50-Gy irradiated tissues stained with H\&E. (A) Bone tissues: a) normal foot bone; b) fibrosis of endosteal and bone marrow tissue containing adipocytes; c) foot bone resorption cavities containing osteoclasts and bone marrow tissue replaced by an amorphous eosinophil substance; d) normal tibia periosteum adherent to the cortical bone; e) blood vessels of the periosteum destroyed and replaced by fibrosis; f) blood vessels of the periosteum destroyed and replaced by fibrosis; g) Howship's lacunae containing osteocytes on the cortical bone; h) some Howship's lacunae are empty; i) empty Howship's lacunae, the death of bone cells results in acellular bone; j) endosteal blood vessels, which supply bone marrow; k) endosteal blood vessels surrounded by fibrosis; 1) endosteal blood vessels destroyed and replaced by fibrosis; m) normal bone marrow tissue containing hematopoietic cells; n) bone marrow tissue containing hematopoietic cells and adipocytes; o) bone marrow tissue totally replaced by adipocytes and surrounded by an amorphous eosinophil substance. (B) Soft tissues: a) normal muscle fiber architecture; b) collagen and inflammatory infiltration between the muscle fibers; c) presence of a fibro-necrotic area with important inflammatory infiltration within the muscle; d) well-vascularized cortical bone; e) narrowing of the vascular lumen; f) loss of intra-osseous vascularization; g) blood vessels; h) decrease in endothelial cells and collagenous hyalinization of the blood vessel wall; i) disappearance of endothelial cells, realignment of the blood vessel wall with narrowing of the vascular lumen. 
A

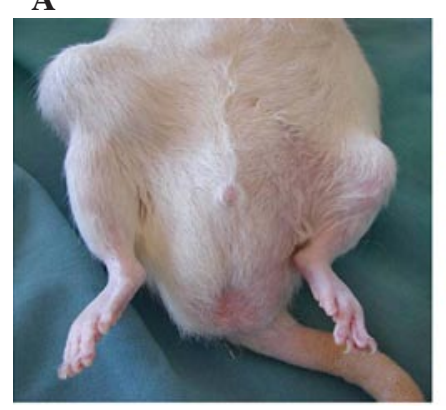

B

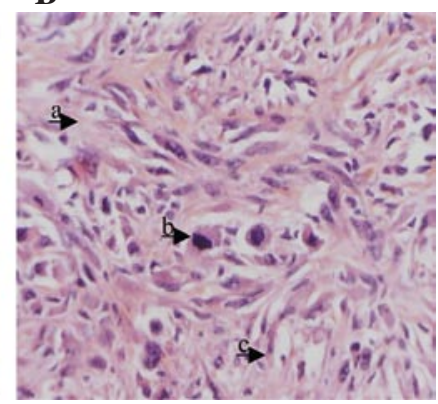

Figure 7. Macroscopic and microscopic images taken from irradiation-induced tumor. a, collagen; b, multinucleted giant cells; c, histiocyte-like cells.

In the present study, changes in the clinical observation records of stage were noted, and in follow-up studies in humans (17), time- and dose-dependent injuries in the irradiated areas were observed. Similar to previous studies (24-26), one well-documented acute adverse effect of irradiation is on the skin. Indeed, alopecia and irradiation-induced dermatitis were noted after exposure of rat hindlimbs to 30 or $50 \mathrm{~Gy}$; this effect was more pronounced in animals which initially received a higher dose. Generally, hindlimb alopecia became apparent after 2 weeks in Group 3 (50 Gy) and on average 4-5 weeks after irradiation with $30 \mathrm{~Gy}$. This delayed effect was consistent with recent findings. In a previous study (26), all rats receiving a single dose of $20 \mathrm{~Gy}$ at a high-dose rate for brachytherapy developed localized alopecia within 2 weeks. Notably, in a study by François et al (25), NOD/SCID mouse hindlimbs were irradiated with 30 Gy using a 60Co source, and an initial skin defect was observed within the first week, characterized by dry desquamation. This developed into severe desquamation during the second week. The observed acute effect may be attributed, in part, to the increased sensitivity of the skin of immuno-depressed mice to irradiation.

In the present study, upon long-term follow-up, the development of ORN was noted in animals that received $50 \mathrm{~Gy}$. The chronic events were first identified as edema on the foot, followed by an aggressive and increased necrosis after 29 weeks. In our model, hindlimb ORN was primarily located in the areas exposed to high strains and began in an area of terminal vascularization. Furthermore, exposed and devitalized bone was observed through a non-healing skin ulceration, similar to previously observed cases of human ORN (15).

In this study, we employed sequential scintigraphy using ${ }^{99 \mathrm{~m} T c-H D P}$ to assess bone blood flow and bone uptake. These radiopharmaceuticals are known to rapidly incorporate into the bone in direct proportion to the bone blood flow and provide a highly sensitive diagnosis of osseous lesions $(4,27)$. In our study, bone blood flow activity was slightly decreased after irradiation of $30 \mathrm{~Gy}$, but did not reach statistical significance. Pitkanen and Hoppewell (1) conducted SPECT examinations at 1 and 7 months, and a rapid and persistent decrease in the bone blood flow in rat irradiated femurs after exposure to radiation at $25 \mathrm{~Gy}$ was noted. This discrepancy may be due to the strain of rat and/or the irradiation source used. By contrast, in animals receiving a dose of $50 \mathrm{~Gy}$, bone scintigrams have revealed a clear and significant $17 \%$ decrease in blood flow activity at 2 months. Similarly, Cutright and
Brady (20) observed a $28 \%$ decreased vascularity in the irradiated rat humerus 2 months after a single dose of $40 \mathrm{~Gy}$, as compared to the controls. In the present study, the effect of irradiation on bone perfusion was consistently documented with scintigraphy before the clinical diagnosis of ORN. The findings are consistent with the current hypothesis that ORN originates from vascular lesions. This explains why the mandible, with its unique pedicle, is particularly sensitive to radiation compared to other exposed bones (28). Notably, according to our SPECT examination conducted at 8 months post-irradiation, there was a $72 \%$ increase in early ${ }^{99 \mathrm{~m} T c-H D P}$ uptake in the 50 Gy-irradiated group compared to the controls. The phenomenon of excessive tracer uptake in ORN was previously reported $(2,4)$, particularly in the ORN sites of patients (27). Various explanations, such as the possible contribution of post-irradiated inflammatory conditions and/or an increase in cell membrane permeability responsible for a leakage of the tracer, may be advanced. Our bone radiographs conducted at 8 months post-irradiation revealed bone lyses, i.e., a significant decrease in the bone mineralization activity, in 50-Gy vs. 30-Gy irradiated bone. This confirmed our data regarding the alteration in bone uptake after exposure to irradiation irrespective of dose (30 or 50 Gy) (Fig. 4B), in agreement with the findings of King et al (29). It should, however, be noted that, despite the increased blood flow during ORN observed here with a dose of $50 \mathrm{~Gy}$, there was no notable enhancement in bone uptake. Thus, the data were contradicted by histopathological findings, which showed a decrease in vascularity. This issue remains unresolved (4).

Our pathological data were satisfactorily consistent with previous studies. Upon the administration of a dose of $30 \mathrm{~Gy}$, there was a decrease in vascularization (20,30). Maeda et al (31) reported that the osteocyte lacuanae of rats became empty after irradiation with a single dose of $35 \mathrm{~Gy}$. We found a partial loss of osteocytes at $30 \mathrm{~Gy}$. According to Jacobsson et al (32) and Sugimoto et al (9), a certain amount of osteocytes remains viable after a single dose of $40 \mathrm{~Gy}$. At $50 \mathrm{~Gy}$, changes in bony tissue involving the loss of all osseous cell types, osteoblasts and osteocytes, occur. The bone marrow was found to be mostly acellular and contained a significant amount of fat and an amorphous eosinophil substance. Indeed, bone marrow is a very radiosensitive tissue. El-Naggar et al (33) found an irreversible reduction in hematopoietic cells after a fractionated irradiation of $50 \mathrm{~Gy}$. Similarly to ORN, there was a decrease in endothelial cells (3-6), periosteal fibrosis (5) and a reduction in the fibrosis of osseous vascularization $(3,17)$. These histopathological findings are consistent with those of a previous human biopsy study (34). Taken together, these injuries are thought to be responsible for progressive alterations in bone structure, leading to the development of hypoxic, hypovascular and hypocellular bony tissue consistent with the 3-H concept of Marx (35). However, the contribution of each event in ORN development remains largely debated $(2,36)$.

Lastly, among the most serious adverse effects of irradiation are the development of tumors. Although little progress has been made recently in quantifying such risks in animal models due to the difficulty in predicting such a late event, warnings about the potential for long-term malignancy after irradiation continue to be issued (30). Here, we observed two sarcomas 10 months after exposure to $50 \mathrm{~Gy}$. The exact 
frequency of this event was not determined due to the limited duration of the study (10 months), since most of the animals that developed concomitant severe ORN were euthanized out of compassion. The exact mechanism of malignancy and/or ORN requires further investigation.

In conclusion, although the irradiation modality of our model is not directly comparable to that used in human treatment, the lesions were very similar to those observed after conventional fractionated radiotherapy. The lesions were dosedependent and, when exposed to $50 \mathrm{~Gy}$, the model was easy and predictable. All data (clinical, imaging and histological) were concordant.

Although this model is perfectible, it is reliable for exploring the pathogenesis of radio-induced tissue degeneration and ORN, and may be used for assessing the efficiency of existing treatments, including hyperbaric oxygenotherapy, antibiotic treatments and reconstructive surgery, or new therapeutic approaches, such as pentoxifylline, tocopherol or biotherapy with mesenchymal stem cells.

\section{Acknowledgements}

This study was supported by the French Ligue Contre le Cancer, Comités Lorrains.

\section{References}

1. Pitkanen MA and Hopewell JW: Functional changes in the vascularity of the irradiated rat femur. Implications for late effects. Acta Radiol Oncol 22: 253-256, 1983.

2. Store G and Granstrom G: Osteoradionecrosis of the mandible: a microradiographic study of cortical bone. Scand J Plast Reconstr Surg Hand Surg 33: 307-314, 1999.

3. Takahashi S, Sugimoto M, Kotoura Y, Sasai K, Oka M and Yamamuro T: Long-term changes in the haversian systems following high-dose irradiation. An ultrastructural and quantitative histomorphological study. J Bone Joint Surg Am 76 722-738, 1994.

4. Bachmann G, Rossler R, Klett R, Rau WS and Bauer R: The role of magnetic resonance imaging and scintigraphy in the diagnosis of pathologic changes of the mandible after radiation therapy. Int J Oral Maxillofac Surg 25: 189-195, 1996.

5. LaRue SM, Wrigley RH and Powers BE: A review of the effects of radiation therapy on bone. Vet Radiol 28: 17-22, 1987.

6. Sugimoto M, Takahashi S, Kotoura Y, et al: Osteocyte viability after high-dose irradiation in the rabbit. Clin Orthop Relat Res 297: 247-252, 1993

7. Travis EL: Organizational response of normal tissues to irradiation. Semin Radiat Oncol 11: 184-196, 2001.

8. Engleman MA, Woloschak G and Small W Jr: Radiation-induced skeletal injury. Cancer Treat Res 128: 155-169, 2006.

9. Sugimoto M, Takahashi S, Toguchida J, Kotoura Y, Shibamoto Y and Yamamuro T: Changes in bone after high-dose irradiation. Biomechanics and histomorphology. J Bone Joint Surg Br 73 : 492-497, 1991.

10. Pitak-Arnnop P, Sader R, Dhanuthai K, et al: Management of osteoradionecrosis of the jaws: an analysis of evidence. Eur J Surg Oncol 34: 1123-1134, 2008.

11. Reuther T, Schuster T, Mende U and Kubler A: Osteoradionecrosis of the jaws as a side effect of radiotherapy of head and neck tumour patients - a report of a thirty year retrospective review. Int J Oral Maxillofac Surg 32: 289-295, 2003.

12. Jereczek-Fossa BA and Orecchia R: Radiotherapy-induced mandibular bone complications. Cancer Treat Rev 28: 65-74, 2002.

13. Guntinas-Lichius O, Wendt W, Buentzel J, et al: Head and neck cancer in germany: a site-specific analysis of survival of the Thuringian Cancer Registration Database. J Cancer Res Clin Oncol 136: 55-63, 2010.
14. Teng MS and Futran ND: Osteoradionecrosis of the mandible. Curr Opin Otolaryngol Head Neck Surg 13: 217-221, 2005.

15. Lyons A and Ghazali N: Osteoradionecrosis of the jaws: current understanding of its pathophysiology and treatment. Br J Oral Maxillofac Surg 46: 653-660, 2008.

16. Mendenhall WM: Mandibular osteoradionecrosis. J Clin Oncol 22: 4867-4868, 2004.

17. Store G and Boysen M: Mandibular osteoradionecrosis: clinical behaviour and diagnostic aspects. Clin Otolaryngol Allied Sci 25: $378-384,2000$.

18. Grimm G: Animal experimental studies on the pathogenesis of radiogenic bone injuries in the mandibles of adult rabbits. II. Histometric data. Dtsch Zahn Mund Kieferheilkd Zentralbl Gesamte 54: 352-362, 1970.

19. King MA, Casarett GW and Weber DA: A study of irradiated bone: I. Histopathologic and physiologic changes. J Nucl Med 20: $1142-1149,1979$

20. Cutright DE and Brady JM: Long-term effects of radiation on the vascularity of rat bone - quantitative measurements with a new technique. Radiat Res 48: 402-408, 1971.

21. Tran N, Poussier S, Franken PR, et al: Feasibility of in vivo dual-energy myocardial SPECT for monitoring the distribution of transplanted cells in relation to the infarction site. Eur J Nucl Med Mol Imaging 33: 709-715, 2006.

22. Hsu HY, Chai CY and Lee MS: Radiation-induced muscle damage in rats after fractionated high-dose irradiation. Radiat Res 149: 482-486, 1998.

23. Stone HB, Coleman CN, Anscher MS and McBride WH: Effects of radiation on normal tissue: consequences and mechanisms. Lancet Oncol 4: 529-536, 2003.

24. Baltalarli B, Bir F, Demirkan N and Abban G: The preventive effect of vitamin D3 on radiation-induced hair toxicity in a rat model. Life Sci 78: 1646-1651, 2006.

25. Francois S, Mouiseddine M, Mathieu N, et al: Human mesenchymal stem cells favour healing of the cutaneous radiation syndrome in a xenogenic transplant model. Ann Hematol 86: $1-8,2007$.

26. Niehoff P, Springer IN, Acil Y, et al: HDR brachytherapy irradiation of the jaw as a new experimental model of radiogenic bone damage. J Craniomaxillofac Surg 36: 203-209, 2008.

27. Hutchison IL, Cullum ID, Langford JA, Jarritt PH, Ell PJ and Harris M: The investigation of osteoradionecrosis of the mandible by ${ }^{99 \mathrm{~m}} \mathrm{Tc}-$ methylene diphosphonate radionuclide bone scans. Br J Oral Maxillofac Surg 28: 143-149, 1990.

28. Bras J, de Jonge HK and van Merkesteyn JP: Osteoradionecrosis of the mandible: pathogenesis. Am J Otolaryngol 11: 244-250, 1990.

29. King MA, Weber DA, Casarett GW, Burgener FA and Corriveau O: A study of irradiated bone. Part ii. Changes in Tc-99m pyrophosphate bone imaging. J Nucl Med 21: 22-30, 1980.

30. Baserga R, Lisco $\mathrm{H}$ and Cater DB: The delayed effects of external gamma irradiation on the bones of rats. Am J Pathol 39: 455-472, 1961

31. Maeda M, Bryant MH, Yamagata M, Li G, Earle JD and Chao EY: Effects of irradiation on cortical bone and their timerelated changes. A biomechanical and histomorphological study. J Bone Joint Surg Am 70: 392-399, 1988.

32. Jacobsson M, Kalebo P, Tjellstrom A and Turesson I: Bone cell viability after irradiation. An enzyme histochemical study. Acta Oncol 26: 463-465, 1987.

33. El-Naggar AM, El-Baz LM, Carsten AL, Chanana AD and Cronkite EP: Radiation-induced damage to blood vessels: a study of dose-effect relationship with time after X-irradiation. Int J Radiat Biol Relat Stud Phys Chem Med 34: 359-366, 1978.

34. Marx RE and Johnson RP: Studies in the radiobiology of osteoradionecrosis and their clinical significance. Oral Surg Oral Med Oral Pathol 64: 379-390, 1987.

35. Marx RE: A new concept in the treatment of osteoradionecrosis. J Oral Maxillofac Surg 41: 351-357, 1983.

36. Gal TJ, Munoz-Antonia T, Muro-Cacho CA and Klotch DW: Radiation effects on osteoblasts in vitro: a potential role in osteoradionecrosis. Arch Otolaryngol Head Neck Surg 126: 1124-1128, 2000 